\title{
Osteomyelitis following Domestic Animal Bites to the Hand: Two Case Reports and Practical Guidelines
}

\author{
Jung Soo Lim ${ }^{1}$, Jin Hwan Byun ${ }^{1}$, Kyung Hee Min ${ }^{1}$, Hye Kyung Lee ${ }^{1}$, Yun Sun Choi ${ }^{2}$ \\ Departments of ${ }^{1}$ Plastic and Reconstructive Surgery and ${ }^{2}$ Radiology, Eulji General Hospital, Eulji University School of Medicine, Seoul, Korea
}

Recently, the number of cases of animal bite wounds has increased significantly in concordance with an increase in the pet population around the world. The authors report two rare cases of osteomyelitis of the phalanx following cat and dog bites. On initial physical examination, signs of a severe infection were observed. Radiographs of both patients showed the presence of osteomyelitis, and in one of the patients, the diagnosis was confirmed with a bone biopsy. After use of empirical antibiotics, intravenous antibiotic therapy that matched the identified bacterium's sensitivity was initiated, and at the same time, secure dressing including debridement was performed to induce secondary healing. In addition, the patients were closely monitored with serial X-rays, and culture and blood test follow-up. One patient fully recovered without sequelae, but the other patient suffered a loss of distal interphalangeal joint motion. When dealing with bite wounds located on the hand, it is important to visit the hospital as soon as possible and receive the appropriate treatment early. Moreover, to prevent severe complications such as osteomyelitis, it is important to administer antibiotic therapy to which the cultured bacteria are sensitive, along with proper wound management and prophylactic antibiotic treatment.

Keywords Bites and stings / Hand injuries / Animals / Wound infection
Correspondence: Kyung Hee Min Department of Plastic and Reconstructive Surgery, Eulji General Hospital, Eulji University School of Medicine, 68 Hangeulbiseong-ro, Nowon-gu, Seoul 01830, Korea

Tel: $+82-2-970-8255$

Fax: +82-2-978-4772

E-mail: mkh797@hanmail.net

Received: 17 May 2016• Revised: 23 Jun $2016 \bullet$ Accepted: 5 Jul 2016

pISSN: 2234-6163 • elSSN: 2234-6171 • https://doi.org/10.5999/aps.2016.43.6.590 • Arch Plast Surg 2016;43:590-594

No potential conflict of interest relevant to this article was reported.

\section{INTRODUCTION}

The incidence of injuries caused by domestic animal bites is increasing with a concurrent increase in the pet population worldwide, and Korea is not an exception to this trend $[1,2]$. In the United States, dog bite injury, the most common domestic animal bite injury, accounts for $80 \%$ to $90 \%$ of all bites, and is followed by cat and human bite injury. It is widely accepted that cat bites account for an additional $5 \%$ to $15 \%$ of animal bite wounds [3]. Bites to the hands and upper extremities account for $18 \%$ to
$68 \%$ of all dog bites, which is a considerable proportion [4]. In Korea, it was reported that bites to the hand and upper extremities comprise $43 \%$ of all dog bite wounds in adults [2]. Among all domestic animal bites, it is known that a bite to the hand has a higher rate of infection than a bite to any other part of the body, which seems to be associated with transmission of contaminants when the tendon glides [5]. Moreover, bite wounds on the hand are related to an increased risk of tenosynovitis, septic arthritis, cellulitis, and osteomyelitis [4]. Therefore, early proper management is important when dealing with bite wounds located on 
the hand. The authors present two cases of osteomyelitis and potential osteomyelitis of the phalanx caused by cat and dog bites, respectively.

\section{CASES}

\section{Case 1}

A female patient aged 57 years visited our clinic 7 days after she was bitten on the middle and distal phalanx of her right third finger by a cat. Before visiting our clinic, the patient received dressing at another local clinic, but did not show any significant improvement.

On initial physical examination, severe swelling, tenderness, redness, and yellowish pus were noted. At that time, incision, drainage, and an intraoperative culture were performed; the culture was conducted to confirm the presence of Pasteurella canis. Cefoperazone sodium was injected as empirical antibiotic treatment ( $1 \mathrm{~g} /$ day). After consultation with the division of infectious disease about Pasteurella canis, the patient was hospitalized so that she could receive IV antibiotic therapy with amoxicillin and clavulanic acid (Augmentin, $1 \mathrm{~g} / 8 \mathrm{hr}$; GlaxoSmithKline plc, London, United Kingdom).

Three weeks following the bite, an erythrocyte sedimentation rate (ESR) of $30 \mathrm{~mm} / \mathrm{hr}$ and a C-reactive protein (CRP) level of $0.33 \mathrm{mg} / \mathrm{dL}$ were noted, and bony destruction and a defect with a suspicious fragment at the third distal interphalangeal (DIP) joint and infectious arthritis and osteomyelitis were iden-

\section{Fig. 1. Case 1: lateral finger radiograph}

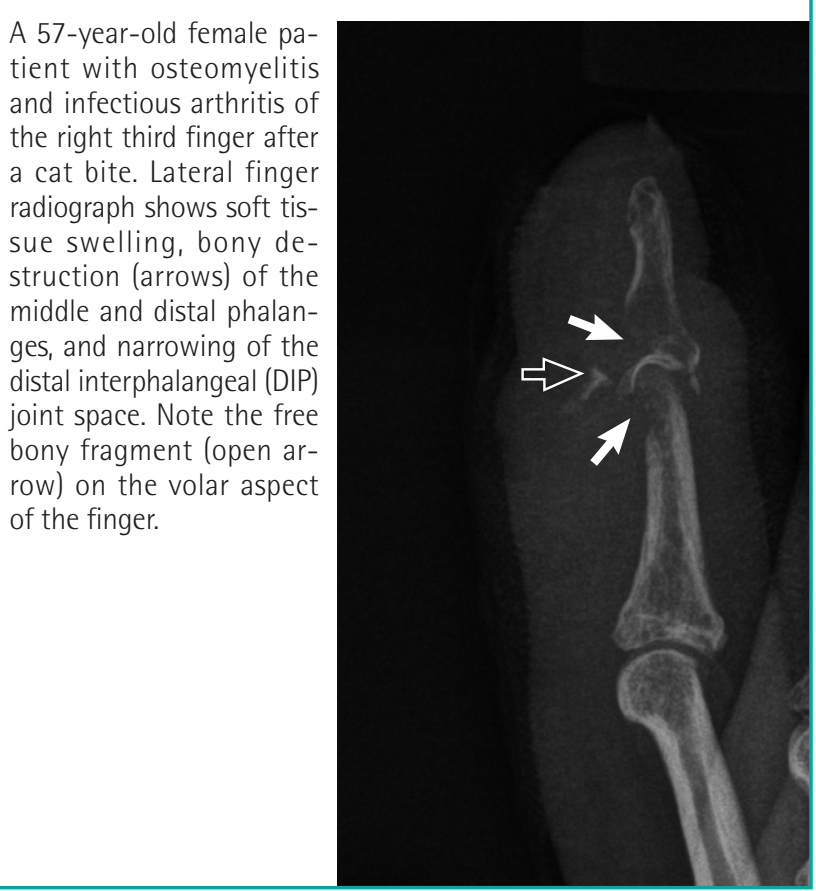

tified on a finger X-ray (Fig. 1). We again consulted with the division of infectious disease about the X-ray findings, and the patient received combination therapy with four antibiotics: moxifloxacin hydrochloride ( $400 \mathrm{mg} /$ day), sulfamethoxazole (400 $\mathrm{mg} / 12 \mathrm{hr}$ ), trimethoprim $(80 \mathrm{mg} / 12 \mathrm{hr}$ ), and clindamycin phosphate $(600 \mathrm{mg} / 8 \mathrm{hr})$.

At approximately one month following the bite, an ESR of 23 $\mathrm{mm} / \mathrm{hr}$ and a CRP level of $0.09 \mathrm{mg} / \mathrm{dL}$ were noted. Discharge, tenderness, and swelling gradually improved and a follow-up culture confirmed negative results.

At approximately six weeks following the bite, an ESR of 32 $\mathrm{mm} / \mathrm{hr}$ and a CRP level of $0.65 \mathrm{mg} / \mathrm{dL}$ were noted. Debridement with bone fragment removal and curettage were performed under digital block. Bone biopsy was performed using the bone fragment, in order to confirm the presence of granulation tissue with acute and chronic inflammation. The suspicious bone fragment was successfully removed and was confirmed on X-ray (Fig. 2). We observed that a segment of a flexor tendon was necrotized and it spontaneously ruptured at the tendon insertion site; hence, we eventually removed the segment, which led to the loss of DIP joint motion. However, with respect to the increase in ESR and CRP values and clinical signs, inflammation and infection were not controlled; therefore, reconstructive surgery was not considered appropriate right away.

At approximately two months following the bite, the wound had healed well. However, loss of DIP joint motion and mild depression at the defect site were observed (Figs. 3, 4). We sug-

\section{Fig. 2. Case 1: lateral finger radiograph follow-up}

On follow-up radiography, soft tissue swelling and bony destruction had improved. The free bony fragment was removed by surgical excision.

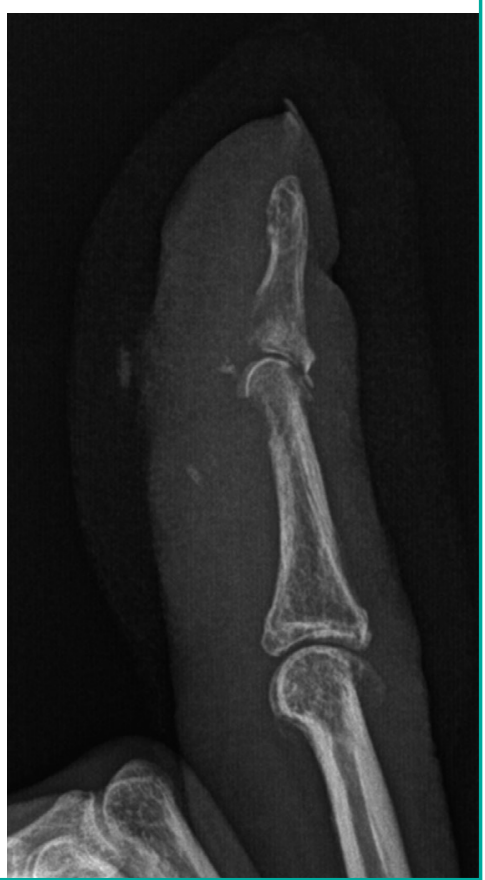




\section{Fig. 3. Case 1: clinical photograph (depression)}

Slight depression in the previous defect site was observed on the volar side of the right third finger.

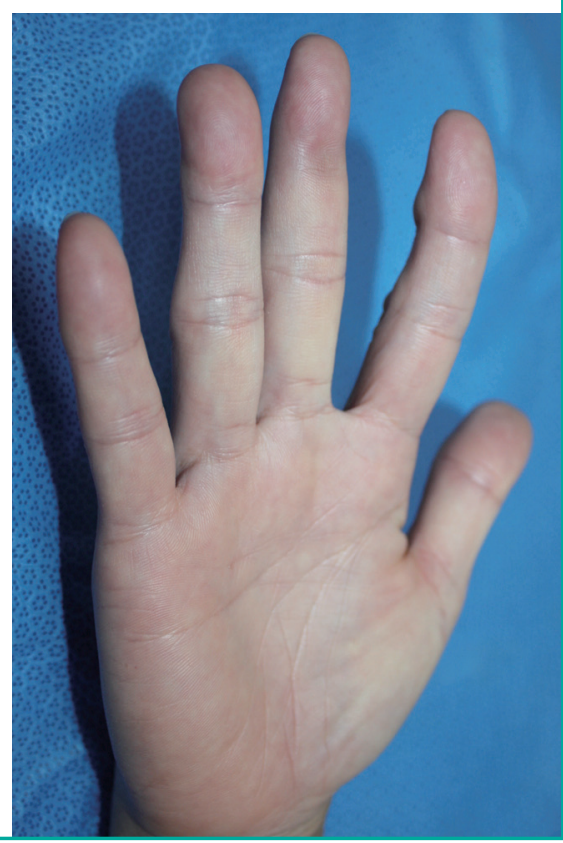

Fig. 4. Case 1: clinical photograph (motion)

Loss of distal interphalangeal joint motion was observed in the right third finger.

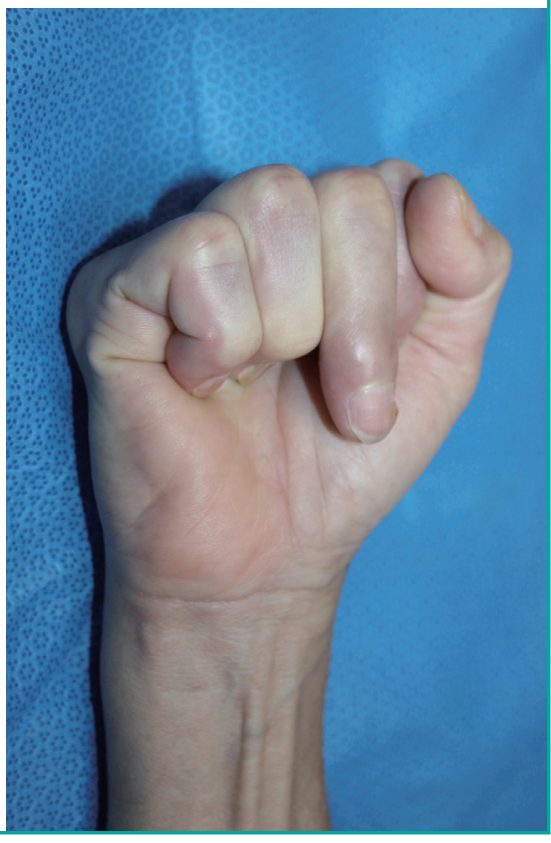

gested that the patient undergo secondary reconstruction with use of a tendon graft, but the patient refused the procedure, and after one year of follow-up, no signs of recurrence were observed.

\section{Case 2}

A female patient aged 52 years visited our clinic 4 days after she was bitten on the distal phalanx of her left third finger by a dog. Before visiting our clinic, the patient took an anti-inflammatory
Fig. 5. Case 2: anteroposterior finger radiograph

A 52-year-old female patient with potential osteomyelitis of the left third finger after a dog bite. An anteroposterior finger radiograph shows soft tissue swelling and slight bony destruction (arrow) on the ulnar side of the distal phalanx.

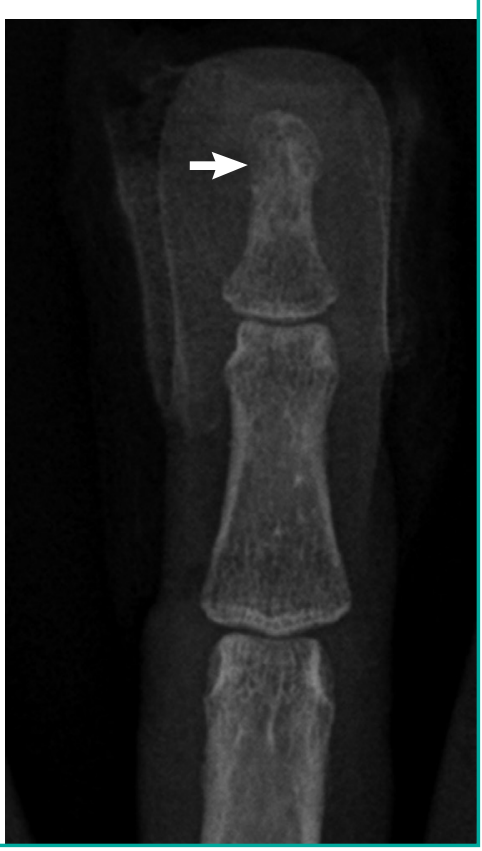

drug orally that was prescribed by the pharmacist at a pharmacy for the first 2 days.

On initial physical examination, purulent discharge from the ulnar aspect of the distal phalanx, along with tenderness and erythema were noted. Radiographs taken at that time revealed soft tissue swelling with no bony abnormality. A culture was performed, which confirmed the presence of many Pseudomonas aeruginosa isolates. The patient was treated with irrigation, dressing twice daily, and intravenous empirical antibiotics (Ceftriaxone, $2 \mathrm{~g}$ /day) for 6 days. After we received the reports of the culture, antibiotics were switched to intravenous ciprofloxa$\operatorname{cin}(200 \mathrm{mg} /$ day).

At approximately two weeks following the bite, and after consultation with the division of infectious disease about Pseudomonas aeruginosa, the patient was hospitalized so that she could receive IV antibiotic therapy with tazobactam and piperacillin sodium ( $4.5 \mathrm{~g} / 8 \mathrm{hr}$ ). At that time, an ESR of $2 \mathrm{~mm} / \mathrm{hr}$ and a CRP level of $0.18 \mathrm{mg} / \mathrm{dL}$ were noted, and osteomyelitis of the ulnar side of the left 3 rd distal phalanx was identified on finger X-ray (Fig. 5). A culture was performed, which showed rare Pseudomonas aeruginosa isolates. We continued IV antibiotic therapy for two more weeks.

At approximately one month following the bite, a follow-up culture confirmed negative results. However, there was no interval change on the finger X-ray. The patient was switched to oral ciprofloxacin $(750 \mathrm{mg} / 12 \mathrm{hr}$ ) based on consultation with the division of infectious disease, and she was discharged.

At approximately 2 months following the bite, improvement 


\section{Fig. 6. Case 2: anteroposterior finger radiograph follow-up}

On follow-up radiograph, soft tissue swelling and bony destruction had healed.

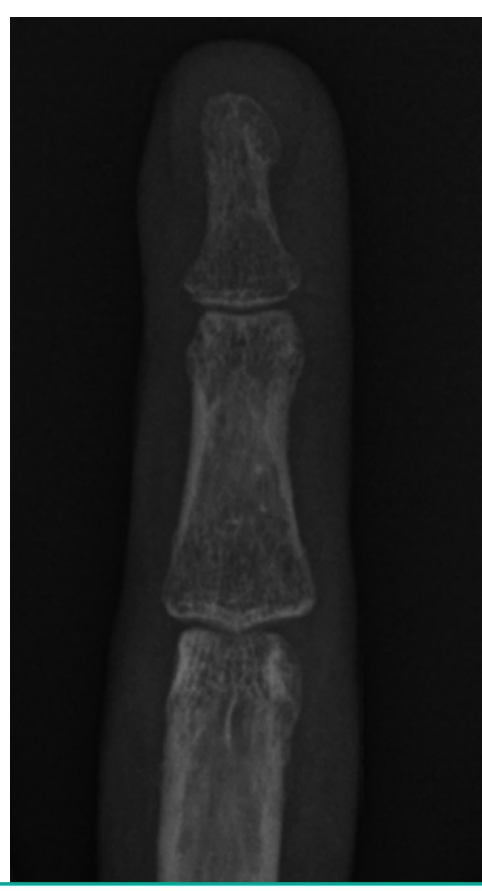

in the left third distal phalanx since the prior study was confirmed on finger X-ray (Fig. 6). Erythema and pain improved without sequelae.

Although we did not confirm the presence of osteomyelitis with bone biopsy and equipment like magnetic resonance imaging (MRI) for further radiological evaluation, we diagnosed osteomyelitis based on plain X-ray interpretation for the following reasons: Firstly, the site of bite that showed clinical signs was exactly identical to the site in which slight bony destruction was observed on the radiograph, and secondly, we continuously observed the an ongoing correlation between the clinical signs and the radiographs through serial X-ray follow-up. As a result, the lesion in which destruction was observed showed signs of improvement on the radiograph along with alleviation of clinical signs.

\section{DISCUSSION}

Although the incidence of domestic animal bites is showing an increasing trend, it seems to have been underestimated because not all patients visit the hospital $[1,3]$. In general, patients think that severe bite wounds are caused only by huge and aggressive dogs that inflict multiple critical lacerations. However, a single bite puncture from a tiny and nonthreatening pet is often underestimated and not treated properly, and eventually may require a surgical procedure for deep infection and result in permanent residual impairment [1].
In our two patients with single bite puncture open wounds, a domestic cat and a tiny Maltese dog caused the injury, respectively. There was no history of a visit to the emergency department for either patient, and they delayed their visit to our clinic by 7 and 4 days, respectively. Symptoms of cellulitis were already present in both patients upon arrival at our clinic.

Bites to the hands and upper extremities account for $18 \%$ to $68 \%$ of all dog bites, which is a considerable proportion [4]. Similarly, Kim et al. [2] reported that bites to the hands and upper extremities comprise $43 \%$ of all dog bite wounds among adults in Korea specifically.

It is a well-known fact that the infection rate of hand bites is high $[1,4,5]$. Furthermore, injury to these areas has the potential to produce severe sequelae such as tenosynovitis, septic arthritis, and osteomyelitis $[1,4]$.

Unlike cellulitis, osteomyelitis is a rare complication after dog and cat bites. However, there is a distinct possibility that cellulitis will progress to osteomyelitis of the hand and upper extremity, and can be observed in the areas where the bones and tendons are close to the surface $[6,7]$.

The relative rarity of osteomyelitis following dog bites compared with cat bites is probably due to the differences in the major mechanisms of injury. While cats tend to inflict deep, penetrating puncture wounds that seal off almost immediately, dog bites tend to be a tearing type of injury, which often allows for free drainage $[1,4,8,9]$. Hence, the infection rate for cat bites is higher than that for dog bites, as documented in prior studies $[1,10,11]$.

The principle of treatment in domestic animal bites to the hand is that wounds are considered grossly contaminated. Firstly, radiography is performed if presence of a foreign body or bone involvement is suspected $[5,9,10]$. As seen in our cases, osteomyelitis after animal bites could not be initially identified on X-ray, and the infection progressed to osteomyelitis as identified by $\mathrm{X}$ ray later. Therefore, serial follow-up radiographs should be performed in cases of animal bites to the hand.

According to the current guidelines by prior studies, the principle of wound management in domestic animal bites to the hand is as follows: The puncture site should be left open to allow for drainage, and copious high-pressure irrigation and cautious debridement of the devitalized tissue should be performed, with careful exploration of tendon or bone involvement and foreign bodies $[1,4,8-10]$.

There are contradictory data regarding antibiotic prophylaxis in the treatment of domestic animal bite wounds. However, prophylactic antibiotics should be recommended for domestic animal bites to the hand according to the current guidelines by previous studies $[5,9,10]$. 
In addition, amoxicillin/clavulanate (Augmentin) is generally believed to be the first-line prophylactic treatment for domestic animal bites before confirmation by the reports of culturing [11, 12]. After confirmation by the culture reports, antibiotics to which the cultured bacteria are sensitive should be employed. In our cases, both patients received proper antibiotic treatments sensitive to cultured bacteria, which had been confirmed to be Pasteurella canis and Pseudomonas aeruginosa, respectively.

In 2002, Hara et al. [6] reported osteomyelitis of the hand by Pasteurella canis, which is a rare type of bacterium in domestic animal bites, for the first time. Pasteurella canis is also known as an opportunistic bacterium. The patient reported by Hara was an elderly man with diabetes, while our patient was a healthy person without any possibility of opportunistic infection. To the best of our knowledge, our case may be the first report of Pasteurella canis osteomyelitis in an otherwise healthy human without any possibility of opportunistic infection.

In addition, tetanus vaccination is suggested after a domestic animal bite if more than 5 years has elapsed since the patient had been immunized [13].

There is an important similarity between our cases; both patients had a delay in their visit to our clinic from the time of injury. As a result, they did not receive appropriate early treatment for bite wounds. Bite wound infections in both patients progressed to osteomyelitis, and in one of the patients, the diagnosis was confirmed by bone biopsy. Eventually, a sequela, specifically, loss of DIP joint motion, was observed in one of these patients.

Based on our experience in these two cases and a review of a number of previous studies, we recommend the following practical guidelines for domestic animal bites to the hand.

(1) It is important to visit the hospital at the time of injury, and receive appropriate early management.

(2) Amoxicillin/clavulanate (Augmentin) is recommended as the first-line empirical antibiotic in patients. After confirmation by the reports of culture, single or combination IV antibiotics to which the cultured bacteria are sensitive should be employed.

(3) Massive irrigation and cautious debridement should be performed, and the puncture site should be left open to allow drainage to prevent bone and joint infection.

(4) Initial evaluation with an X-ray is helpful for making the diagnosis of a foreign body or bony involvement.

(5) Serial culture, radiographs, and a blood test (for ESR and
CRP) are helpful for determining the prognosis.

(6) Tetanus vaccination is suggested if needed.

(7) Immobilization of the joint is useful for prevention of transmission of contaminants by tendon gliding and damage to the tendon itself.

We expect that physicians will use our case reports and practical guidelines as a proper treatment protocol for hand bites caused by domestic animals.

\section{REFERENCES}

1. Benson LS, Edwards SL, Schiff AP, et al. Dog and cat bites to the hand: treatment and cost assessment. J Hand Surg Am 2006;31:468-73.

2. Kim SH, You JY, Ryu JY. A comparison of characteristics in dog bite patients. J Korean Soc Traumatol 2005;18:135-40.

3. Griego RD, Rosen T, Orengo IF, et al. Dog, cat, and human bites: a review. J Am Acad Dermatol 1995;33:1019-29.

4. Goldstein EJ. Bite wounds and infection. Clin Infect Dis 1992;14:633-8.

5. Lee YG, Jeong SH, Kim WK. An analytical study of Mammalian bite wounds requiring inpatient management. Arch Plast Surg 2013;40:705-10.

6. Hara H, Ochiai T, Morishima T, et al. Pasteurella canis osteomyelitis and cutaneous abscess after a domestic dog bite. J Am Acad Dermatol 2002;46:S151-2.

7. Jarvis WR, Banko S, Snyder E, et al. Pasteurella multocida: osteomyelitis following dog bites. Am J Dis Child 1981;135: 625-7.

8. Desai SS, Groves RJ, Glew R. Subacute Pasteurella osteomyelitis of the hand following dog bite. Orthopedics 1990; 13:653-6.

9. Ellis R, Ellis C. Dog and cat bites. Am Fam Physician 2014; 90:239-43.

10. Oehler RL, Velez AP, Mizrachi M, et al. Bite-related and septic syndromes caused by cats and dogs. Lancet Infect Dis 2009;9:439-47.

11. Morgan M, Palmer J. Dog bites. BMJ 2007;334:413-7.

12. Stevens DL, Bisno AL, Chambers HF, et al. Practice guidelines for the diagnosis and management of skin and soft-tissue infections. Clin Infect Dis 2005;41:1373-406.

13. Fleisher GR. The management of bite wounds. N Engl J Med 1999;340:138-40. 